\title{
REPRESENTATIONS FOR REAL NUMBERS
}

\section{J. EVERETT 1}

1. Introduction. In a recent paper ${ }^{2}$ [1] B. H. Bissinger generalized continued fractions by iteration of more general decreasing functions than the $1 / x$ of the classical case. We extend here the algorithm by which real numbers are represented as decimals of base $p$, to general continuous increasing functions on $(0, p)$, including the classical $x / p$ as special case. This sets up a correspondence from real numbers to sequences of integers $\bmod p$. Weak sufficient conditions are given that the correspondence be one-one. In the one-one case, algebraic examples are noted. The limit involved in the inscribed polygon problem appears here in a natural way. In the many-one case, the algorithm defines a set $L$ of limit numbers which is perfect and nowhere dense. These sets are closely related to the Cantor perfect set. Finally, the relation between the above theory and the topological transformations $F_{1}$ of the unit interval into itself is studied. The latter yield sequences $\left\{F_{p}\right\}$ of our functions, $p=2,3, \cdots$, and their structure is reflected in the limit sets $L_{2}, L_{3}, \cdots$.

2. The algorithm. Let $p \geqq 2$ be a fixed integer and $f(t)$ a continuous, strictly increasing function on the interval $0 \leqq t \leqq p$, with $f(0)=0$ and $f(p)=1$ (cf. [4]).

Such a function may be used to associate with every real number $\gamma_{0} \geqq 0$, a sequence $\left\{c_{\nu}\right\}$ of integers, with $0 \leqq c_{0}<\infty, 0 \leqq c_{\nu} \leqq p-1$, $\nu=1,2, \cdots$, by way of the following algorithm. We write, for $\gamma_{0} \geqq 0$,

$$
\begin{aligned}
& \gamma_{0}=c_{0}+f\left(\gamma_{1}\right), \quad c_{0} \leqq \gamma_{0}<c_{0}+1, \\
& 0 \leqq c_{0}<\infty, 0 \leqq \gamma_{1}<p, \\
& \gamma_{1}=c_{1}+f\left(\gamma_{2}\right), \quad c_{1} \leqq \gamma_{1}<c_{1}+1, \\
& 0 \leqq c_{1} \leqq p-1,0 \leqq \gamma_{2}<p,
\end{aligned}
$$

and so on.

Thus, at each step, $c_{\nu}$ is the greatest integer in $\gamma_{\nu}$, and $\gamma_{v+1}$ is the uniquely defined real number on the interval $0 \leqq t<p$ such that $f\left(\gamma_{\nu+1}\right)=\gamma_{\nu}-c_{\nu}$, where $0 \leqq \gamma_{\nu}-c_{\nu}<1$.

Presented to the Society, September 17,1945 ; received by the editors July 10 , 1945, and, in revised form, April 9, 1946.

1 The work here reported was supported by the Wisconsin Alumni Research Foundation.

${ }^{2}$ The numbers in brackets refer to the references cited at the end of the paper. 
Since $\gamma_{1}, \gamma_{2}, \cdots$ are all on $0 \leqq t<p$, it follows that $c_{1}, c_{2}, \cdots$ are integers satisfying $0 \leqq c_{\nu} \leqq p-1$. Hence we have a correspondence

$$
\gamma_{0} \rightarrow\left\{c_{\nu}\right\}
$$

from all reals $\gamma_{0} \geqq 0$, to sequences of integers as described.

3. Termination in $p-1$. We ask now whether, under the algorithm, sequences may appear terminating in $p-1, p-1, \cdots$. Such is the case if and only if, for the function $f(t)$ :

(C) There exists a $\gamma_{0}=p-1+f\left(\gamma_{0}\right), p-1<\gamma_{0}<p$.

Obviously such a $\gamma_{0} \rightarrow\{p-1, p-1, \cdots\}$ under (A). On the other hand, if a number $\delta_{0}$ under (A) yields a sequence terminating in $p-1, p-1, \cdots$, this implies that some $\delta_{\nu}$ itself yields $p-1, p-1, \cdots$. Suppose then that $\gamma_{0}\left(=\delta_{v}\right)$ under (A) gives $\gamma_{0}=p-1+f\left(\gamma_{1}\right)$, $\gamma_{1}=p-1+f\left(\gamma_{2}\right), \cdots, \gamma_{\nu}=p-1+f\left(\gamma_{\nu+1}\right)$, and so on. If (C) is false, it follows from continuity of $f(t)$ that:

$$
f(t)>t-(p-1), \quad \text { for all } t \text { on } p-1 \leqq t<p .
$$

We should then have $\gamma_{1}-(p-1)<f\left(\gamma_{1}\right)=\gamma_{0}-(p-1), \gamma_{2}-(p-1)$ $<f\left(\gamma_{2}\right)=\gamma_{1}-(p-1)$, and so on, with $p>\gamma_{0}>\gamma_{1}>\gamma_{2}>\cdots>p-1$. Hence $t_{0}=\lim \gamma_{\nu}$ exists, with $p-1 \leqq t_{0}<p$. But from $\gamma_{\nu}=p-1+f\left(\gamma_{\nu+1}\right)$, we have $t_{0}=p-1+f\left(t_{0}\right)$, a contradiction.

Indeed, the condition "not $C$ " is equivalent to (D), and (D) may in turn be rephrased as a slope condition

$$
(f(p)-f(t)) /(p-t)<1 \text { on } p-1 \leqq t<p .
$$

Moreover, if a $\gamma_{0}$ satisfying (C) exists, then not only $\gamma_{0}$ but every $\delta_{0}$ on $\gamma_{0}<\delta_{0}<p$ will yield $\{p-1, p-1, \cdots\}$ under (A). For $\gamma_{0}=p-1$ $+f\left(\gamma_{0}\right)<\delta_{0}<p$ implies $\delta_{0}=p-1+f\left(\delta_{1}\right)$, hence $\gamma_{0}<\delta_{1}<p$, and so on. Since our final object is to obtain a one-one correspondence (B), we assume from this point on the necessary condition $\left(D^{\prime}\right)$. The correspondence (B) then maps all reals $\gamma_{0} \geqq 0$ onto non- $(p-1)$-terminating sequences.

4. Upper and lower limits. Let $\left\{c_{\nu}\right\}$ be an arbitrary sequence of integers with $0 \leqq c_{0} \leqq \infty ; 0 \leqq c_{\nu} \leqq p-1, \nu=1,2, \cdots$, not $(p-1)$-terminating. We define $C_{\nu}{ }^{\lambda}=c_{\lambda}+f\left(c_{\lambda+1}+\cdots+f\left(c_{\lambda+\nu}\right)\right.$ and $\Gamma_{\nu}{ }^{\lambda}=c_{\lambda}$ $+f\left(c_{\lambda+1}+\cdots+f\left(c_{\lambda+\nu}+1\right)\right.$, where the last parentheses are $\nu$-fold. Then, from monotonicity, one has $c_{\lambda} \leqq C_{\nu}{ }^{\lambda} \leqq C_{\nu+1}{ }^{\lambda}<\Gamma_{\nu+1}{ }^{\lambda} \leqq \Gamma_{\nu}{ }^{\lambda} \leqq c_{\lambda}+1$, so that the $\operatorname{limits} C^{\lambda}=\lim C_{\nu}{ }^{\lambda}, \Gamma^{\lambda}=\lim \Gamma_{\nu}{ }^{\lambda}$ exist and satisfy

$$
c_{\lambda} \leqq C^{\lambda} \leqq \Gamma^{\lambda} \leqq c_{\lambda}+1 \text {. }
$$

Since $C_{\nu}{ }^{\lambda}=c_{\lambda}+f\left(C_{\nu-1}{ }^{\lambda+1}\right)$ we have $C^{\lambda}=c_{\lambda}+f\left(C^{\lambda+1}\right)$ and similarly 
$\Gamma^{\lambda}=c_{\lambda}+f\left(\Gamma^{\lambda+1}\right)$. Now since the sequence is not $(p-1)$-terminating, for every $\lambda$ there is a $c_{\lambda+\mu} \leqq p-2$. Moreover, $\Gamma_{\mu+\nu}{ }^{\lambda}=c_{\lambda}+f\left(c_{\lambda+1}+\cdots\right.$ $+f\left(\Gamma_{\nu}{ }^{\lambda+\mu}\right)$, and $\Gamma^{\lambda}=c_{\lambda}+f\left(c_{\lambda+1}+\cdots+f\left(\Gamma^{\lambda+\mu}\right)\right.$. By $(\mathrm{E}), \Gamma^{\lambda+\mu} \leqq c_{\lambda+\mu}$ $+1 \leqq p-1$, so that we have

$$
c_{\lambda} \leqq C^{\lambda} \leqq \Gamma^{\lambda}<c_{\lambda}+1,
$$

and, as already shown,

$$
C^{\lambda}=c_{\lambda}+f\left(C^{\lambda+1}\right), \quad \Gamma^{\lambda}=c_{\lambda}+f\left(\Gamma^{\lambda+1}\right) .
$$

But $\left(\mathrm{E}^{\prime}, \mathrm{F}\right)$ imply that, under (A), the numbers $C^{0}$ and $\Gamma^{0}$ yield the original sequence $\left\{c_{\nu}\right\}$. We call these the lower and upper limit numbers of the sequence.

If $f(t)$ satisfies $\left(\mathrm{D}^{\prime}\right)$, the correspondence $(\mathrm{B})$ maps all reals $\gamma_{0} \geqq 0$ onto all non-( $p-1)$-terminating sequences. Every such sequence is indeed the map of its limit numbers $C^{0}, \Gamma^{0}$.

Now if $\gamma_{0}$ yields $\left\{c_{\nu}\right\}$ under (A), then

$$
C_{\nu}^{0} \leqq \gamma_{0}=c_{0}+f\left(c_{1}+\cdots+f\left(c_{\nu}+f\left(\gamma_{\nu+1}\right)<\Gamma_{\nu}^{0}, \quad \text { all } \nu,\right.\right.
$$

and hence $C^{0} \leqq \gamma_{0} \leqq \Gamma^{0}$.

Also, if $\gamma_{0}^{\prime}$ and $\gamma_{0}^{\prime \prime}$ yield $\left\{c_{\nu}\right\}$ under (A), and if $\gamma_{0}^{\prime} \leqq \gamma_{0} \leqq \gamma_{0}^{\prime \prime}$, then $\gamma_{0}$ yields $\left\{c_{\nu}\right\}$. For

$$
c_{0} \leqq \gamma_{0}^{\prime}=c_{0}+f\left(\gamma_{1}^{\prime}\right) \leqq \gamma_{0} \leqq \gamma_{0}^{\prime \prime}=c_{0}+f\left(\gamma_{1}^{\prime}\right)<c_{0}+1,
$$

hence $\gamma_{0}=c_{0}+f\left(\gamma_{1}\right)$ and $\gamma_{1}^{\prime} \leqq \gamma_{1} \leqq \gamma_{1}^{\prime \prime}$, and so on.

It follows that $\gamma_{0}$ yields $\left\{c_{\nu}\right\}$ under (A) if and only if $C^{0} \leqq \gamma_{0} \leqq \Gamma^{0}$. Thus the correspondence (B) is actually a mapping of disjoint closed sets $\left[C^{0}, \Gamma^{0}\right]$ on all non- $(p-1)$-terminating sequences. The sequences $\left\{c_{\nu}\right\}$ fall into two classes according as $C^{0}<\Gamma^{0}$ or $C^{0}=\Gamma^{0}$. The correspondence (B) thus splits into two parts:

$$
\left[C^{0}, \Gamma^{0}\right] \rightarrow\left\{c_{\nu}\right\}, \quad C^{0}<\Gamma^{0},
$$

In the case $\left(\mathrm{B}^{\prime \prime}\right)$ the $C_{\nu}{ }^{0}$ and $\Gamma_{\nu}{ }^{0}$ converge to $C^{0}=\Gamma^{0}=\gamma_{0}$ with errors thus (see G):

$$
0 \leqq \gamma_{0}-C_{\nu}^{0}<\Gamma_{\nu}^{0}-C_{\nu}^{0} ; \quad 0<\Gamma_{\nu}^{0}-\gamma_{0} \leqq \Gamma_{\nu}^{0}-C_{\nu}^{0}
$$

We note here two properties of the sequence $\{p-1, p-1, \cdots\}$ of later use. Although this sequence does not appear under the algorithm, nevertheless the $\lim C_{\nu}{ }^{0}$ exists and is $p$. For $p-1<C_{\nu}{ }^{0}<C_{\nu+1}{ }^{0}$ $<p$ and $t_{0}=\lim C_{\nu}{ }^{0}$ satisfies $p-1<t_{0} \leqq p$. But $C_{\nu}{ }^{0}=p-1+f\left(C_{\nu-1}{ }^{1}\right)$ $=p-1+f\left(C_{\nu-1}\right)$. Hence $t_{0}=p-1+f\left(t_{0}\right)$, and by $\left(\mathrm{D}^{\prime}\right), t_{0}=p$. 
Also, $p-1+f\left(p-1+\cdots+f\left(p-1+f(p-2) \geqq C_{\nu-1}{ }^{0}\right.\right.$ where the first expression contains $\nu(p-1)$ 's. Thus the sequence $p-1+f(p-2)$, $p-1+f(p-1+f(p-2), \cdots$ has limit $p$.

5. Terminating sequences. We call a sequence $\left\{c_{\nu}\right\}$ with $c_{\nu}=0$, $\nu>N$ for some $N$, terminating. There exist numbers $\gamma_{0}>0$ yielding $\{0,0, \cdots\}$ under $(\mathrm{A})$ if and only if $f(t)$ has the property:

(I) There exists a $\gamma_{0}=f\left(\gamma_{0}\right), 0<\gamma_{0}<1$.

Clearly such a $\gamma_{0}$ yields $\{0,0, \ldots\}$ under (A). Suppose that $\gamma_{0}>0$ yields $\{0,0, \cdots\}$ and that (I) is false. By continuity of $f(t)$ we have

$$
f(t)<t \text { for all } t \text { on } 0<t \leqq 1,
$$

and $0<\gamma_{0}=f\left(\gamma_{1}\right)<\gamma_{1}=f\left(\gamma_{2}\right)<\gamma_{2} \ldots$. Hence $0<\gamma_{0}<\gamma_{1}<\gamma_{2}<\ldots$ $<1$, and $t_{0}=\lim \gamma_{\nu}$ exists with $0<t_{0} \leqq 1$. But from $\gamma_{\nu}=f\left(\gamma_{v+1}\right)$ follows $t_{0}=f\left(t_{0}\right)$, a contradiction.

Obviously "not I" is equivalent to $(\mathrm{J})$, and $(\mathrm{J})$ may be restated in slope form

$$
f(t)-f(0) / t<1 \quad \text { on } 0<t \leqq 1 .
$$

If a $\gamma_{0}$ exists satisfying (I) then not only $\gamma_{0}$ but also every $\delta_{0}$ on $0 \leqq \delta_{0}<\gamma_{0}$ will yield $\{0,0, \cdots\}$ under $(\mathrm{A})$. Hence for a one-one correspondence $(B),\left(\mathrm{J}^{\prime}\right)$ is necessary, and we assume from this point on that $f(t)$ satisfies $\left(\mathrm{D}^{\prime}\right)$ and $\left(\mathrm{J}^{\prime}\right)$.

Under these restrictions, the sequence $\{0,0, \cdots\}$ has $C^{0}=\Gamma^{0}=0$, and since in any sequence $\left\{c_{\nu}\right\}, C^{0}=c_{0}+\cdots+f\left(C^{\lambda}\right), \Gamma^{0}=c_{0}+\cdots$ $+f\left(\Gamma^{\lambda}\right)$, it follows that every terminating sequence has $\Gamma^{0}=C^{0}$ and falls under $\left(B^{\prime \prime}\right)$.

We remark here that if $\left\{d_{\nu}\right\}$ is a terminating sequence $\left\{d_{1}, d_{2}, \cdots\right.$, $\left.d_{\nu}, 0,0, \cdots\right\}$, then the associated limit numbers $D^{0}=\Delta^{0}=d_{0}$ $+f\left(d_{1}+\cdots+f\left(d_{\nu}+f\left(D^{\nu+1}\right)=d_{0}+f\left(d_{1}+\cdots+f\left(d_{v}\right)\right.\right.\right.$, since $D^{v+1}=0$.

6. The many-one case. Suppose then that $f(t)$ satisfies $\left(D^{\prime}\right)$ and $\left(\mathrm{J}^{\prime}\right)$ and consider the algorithm (A) only as it applies to numbers $\gamma_{0}$ on the interval $[0, p)=(0 \leqq t<p)$. The correspondences $\left(\mathrm{B}^{\prime}, \mathrm{B}^{\prime \prime}\right)$ then map the interval $[0, p)$ onto all non- $(p-1)$-terminating sequences $\left\{c_{\nu}\right\}$ with $0 \leqq c_{\nu} \leqq p-1$.

Let $L$ be the set of all limit numbers $C^{0}, \Gamma^{0}$ on $[0, p)$ (equal or not) of all such sequences, and $G$ the complement of $L$ in $[0, p)$. The points of $L$ are then the numbers $C^{0}=\Gamma^{0}$ occurring under $\left(\mathrm{B}^{\prime \prime}\right)$, including the limits of all terminating sequences, together with the end points $C^{0}<\Gamma^{0}$ of the closed intervals under $\left(\mathrm{B}^{\prime}\right)$. The points of $G$ are those of all the open intervals $\left(C^{0}, \Gamma^{0}\right)$ in $\left(\mathrm{B}^{\prime}\right)$. Since $G$ is a union of (non- 
overlapping, indeed, non-abutting) intervals, $G$ is open, and $L$ is closed.

We write $[0, p)=L+G$, and $L=L^{\prime}+L^{\prime \prime}$, where $L^{\prime}$ is the set of end points under $\left(\mathrm{B}^{\prime}\right)$ and $L^{\prime \prime}$ the set of $C^{0}=\Gamma^{0}$ under $\left(\mathrm{B}^{\prime \prime}\right)$.

Since the intervals of $G$ are countable, so is the set $L^{\prime}$. We now show that $L$ is dense in itself. It then follows that $L$ is perfect, $L$ (hence also $\left.L^{\prime \prime}\right)$ has the power of the continuum. Since the limits of terminating sequences are countable, the set of limits of non-terminating sequences for which $C^{0}=\Gamma^{0}$ is of the power of the continuum (cf. [3]).

Indeed, every point $\lambda$ of $L$ is a limit point of limit numbers $D^{0}=\Delta^{0}$ of terminating sequences $\left\{d_{\nu}\right\}$. First let $\lambda=C^{0}=\Gamma^{0}$ for $\left\{c_{\nu}\right\}$. Then $\lambda=\lim C_{\nu}{ }^{0}=\lim \Gamma_{\nu}{ }^{0}$ and $C_{\nu}{ }^{0}<\Gamma_{\nu}{ }^{0}$. The numbers $C_{\nu}{ }^{0}$ are in $L$, being limit numbers of terminating sequences. Since the sequence $\left\{c_{\nu}\right\}$ is not $(p-1)$-terminating, a subsequence of $\left\{\Gamma_{\nu}{ }^{0}\right\}$ has $\Gamma_{\nu}{ }^{0}=c_{0}+f\left(c_{1}+\cdots\right.$ $+f\left(c_{\nu}+1\right)$ with $c_{\nu}+1 \leqq p-1$, and these $\Gamma_{\nu}{ }^{0}$ are thus in $L$, being limit numbers of terminating sequences $\left\{c_{0}, c_{1}, \cdots, c_{\nu}+1,0,0, \cdots\right\}$ in our class. Hence $\lambda$ is a limit point of points of $L$.

Second, let $\lambda=C^{0}<\Gamma^{0}$ for $\left\{c_{\nu}\right\}$. Then the sequence $\left\{c_{\nu}\right\}$ is not terminating, and a subsequence of $\left\{C_{\nu}{ }^{0}\right\}$ is properly increasing to $C^{0}$ as a limit point.

Finally, let $C^{0}<\Gamma^{0}=\lambda$ for $\left\{c_{\nu}\right\}$. Since $\left\{c_{\nu}\right\}$ is not $(p-1)$-terminating, a proper subsequence of $\left\{\Gamma_{\nu}{ }^{0}\right\}$ with $c_{\nu}+1 \leqq p-1$ is properly decreasing to $\Gamma^{0}$ as a limit point. Hence $L$ is dense in itself.

If $f(t)$ admits a sequence $\left\{d_{\nu}\right\}$ with $D^{0}<\Delta^{0}$, that is, if the correspondence (B) is not one-one, then the set $L$ is nondense on $[0, p)$. If $(a, b)$ is a subinterval: $0 \leqq a<b<p$, we show that $(a, b)$ contains a subinterval containing no point of $L$. If $(a, b)$ itself contains no point of $L$, $(a, b)$ will serve. However if a point $\lambda$ of $L$ is in $(a, b)$ and if $\lambda=C^{0}<\Gamma^{0}$ or $C^{0}<\Gamma^{0}=\lambda$ for some $\left\{c_{\nu}\right\}$ then the interval $(a, b)$ intersects $\left(C^{0}, \Gamma^{0}\right)$ in an interval containing only points of $G$. The only case remaining is $\lambda=C^{0}=\Gamma^{0}$ in $(a, b), \lambda=\lim C_{\nu}{ }^{0}=\lim \Gamma_{\nu}{ }^{0}$. But $C_{\nu}{ }^{0}<c_{0}+f\left(c_{1}+\ldots\right.$ $+f\left(c_{\nu}+f\left(D^{0}\right)<c_{0}+f\left(c_{1}+\cdots+f\left(c_{\nu}+f\left(\Delta^{0}\right)<\Gamma_{\nu}{ }^{0}\right.\right.\right.$. The inner numbers define an interval of $G$, interior to $(a, b)$ for sufficiently large $\nu$.

7. An example. Consider for $p=3$ the function $f(t)$ defined by $f(0)=0, f(4 / 3)=1 / 3, f(5 / 3)=2 / 3, f(3)=1$, and elsewhere by the broken line connecting these points. It is clear that $4 / 3$ and $5 / 3$ yield $\{1,1, \cdots\}$ under the algorithm. Moreover, for this sequence, $C^{0}=4 / 3$ and $\Gamma^{0}=5 / 3$ as is seen graphically from the sequences $1+f(1), 1+f(1+f(1), \cdots$ and $1+f(2), 1+f(1+f(2), \cdots$ Imagine that we blacken the intervals $\left(i+f\left(C^{0}\right), i+f\left(\Gamma^{0}\right)\right), i=0,1,2$. The first of these defines three intervals $\left(j+f\left(0+f\left(C^{0}\right), j+f\left(0+f\left(\Gamma^{0}\right)\right), j=0,1\right.\right.$, 2 , and the last similarly, all of which we blacken. (Graphically, the 
process amounts to projecting the function values above each black interval onto the three $45^{\circ}$ lines and thence down to the $t$-axis.) Repetition of this process yields a set of open intervals of total length $1 / 3+2 / 3+3(2 / 3)(1 / 4)+\cdots=1 / 3+2 / 3\left(1+3 / 4+(3 / 4)^{2}+\cdots\right)=3$. It follows that the set of black intervals exhausts the set $G$, and the complement $L$ is of measure zero, perfect, and nondense on $[0,3)$. While this is not quite the Cantor "middle-third" set it has precisely the same structure.

8. Sufficient conditions for one-one correspondence. Let $c_{0}<\gamma_{0}<\delta_{0}$ $<c_{0}+1$ and $\gamma_{0}, \gamma_{1}, \cdots, \gamma_{n} ; \delta_{0}, \delta_{1}, \cdots, \delta_{n}$, be the numbers resulting from the first $n$ steps of the algorithm. We say that the slopes $f\left(\delta_{i}\right)-f\left(\gamma_{i}\right) / \delta_{i}-\gamma_{i}, i=1, \cdots, n$, are connected.

In order that the correspondence (B) be one-one it is sufficient that:

(K) There exists an integer $n$ such that the product of every $n$ connected slopes is less than one.

Suppose that (B) is not one-one, and let $X^{\prime}$ be the class of all intervals $\left(C^{0}, \Gamma^{0}\right)$ under $\left(B^{\prime}\right)$. Then there must be in $X^{\prime}$ an interval of maximal length. For this interval, write $\Gamma^{0}-C^{0}=\left(f\left(\Gamma^{1}\right)-f\left(C^{1}\right) / \Gamma^{1}-C^{1}\right)$ $\cdots\left(f\left(\Gamma^{n}\right)-f\left(C^{n}\right) / \Gamma^{n}-C^{n}\right)\left(\Gamma^{n}-C^{n}\right)$. The interval $\left(C^{n}, \Gamma^{n}\right)$ is in $X^{\prime}$, hence these $n$ connected slopes have product not less than 1, contradicting $(\mathrm{K})$.

Stronger sufficient conditions are:

$$
f\left(t_{2}\right)-f\left(t_{1}\right) / t_{2}-t_{1}<1, \quad 0 \leqq t_{1}<t_{2} \leqq p .
$$

$\left(\mathrm{K}^{\prime \prime}\right)$ There exists a $\beta$ such that $0<\beta<1, f\left(t_{2}\right)-f\left(t_{1}\right) / t_{2}-t_{1} \leqq \beta$ on $0 \leqq t_{1}<t_{2} \leqq p$.

In case $\left(\mathrm{K}^{\prime \prime}\right)$ obtains, we note that $\Gamma_{\nu}{ }^{0}-C_{\nu}{ }^{0}=\left(f\left(\Gamma_{\nu-1}{ }^{1}\right)-f\left(C_{\nu-1}{ }^{1}\right)\right) / \Gamma_{\nu-1}{ }^{1}$ $\left.-C_{\nu-1}\right) \cdots\left(f\left(\Gamma_{1}{ }^{\nu-1}\right)-f\left(C_{1}{ }^{\nu-1}\right) / \Gamma_{1}^{\nu-1}-C_{1}^{\nu-1}\right)\left(f\left(c_{\nu}+1\right)-f\left(c_{\nu}\right) /\left(c_{\nu}+1\right.\right.$ $\left.-c_{\nu}\right) \leqq \beta^{\nu}$, so that from (H) the error in the $\Gamma_{\nu}{ }^{0}$ and $C_{\nu}{ }^{0}$ approximations to $\gamma_{0}=C^{0}=\Gamma^{0}$ is not greater than $\beta^{p}$.

Although the slope condition $\left(\mathrm{K}^{\prime}\right)$ is sufficient for one-one (B), it is far from necessary. We shall construct functions of arbitrarily great slope for which (B) is one-one.

Consider the set of all ratios (note: not slopes) $f(b+f(a+1)$ $-f(b+f(a)) / f(b+1)-f(b)$ where $a, b$ are arbitrary integers on $0,1, \cdots, p-1$. Of these there are only a finite number, each less than one, since the numerator is the difference of function values on a proper subinterval of $(b, b+1)$. Let $M$ be the maximum of these ratios, $M<1$.

Now consider the intervals $(b+f(a), b+f(a+1))$ and suppose that the ratio of inner to outer slope of $f(t)$ on each of these intervals is bounded above from $1 / M$, that is: 
(L) There exists a $k<1 / M$ such that

$$
\left(f\left(t_{2}\right)-f\left(t_{1}\right) / t_{2}-t_{1}\right) /(f(b+f(a+1)-f(b+f(a) / f(a+1)-f(a)) \leqq k
$$

for all $t_{1}, t_{2}$ on

$$
b+f(a) \leqq t_{1}<t_{2} \leqq b+f(a+1),
$$

or equivalently:

$\left(\mathrm{L}^{\prime}\right)$ There exists a $k<1 / M$ such that whenever

$$
b+f(a) \leqq t_{1}<t_{2} \leqq b+f(a+1) \text { and } t_{2}-t_{1} \leqq \tau \cdot(b+f(a+1)-(b+f(a)) \text {, }
$$

we must have $f\left(t_{2}\right)-f\left(t_{1}\right) \leqq k \tau(f(b+f(a+1)-f(b+f(a))$.

The condition $\left(\mathrm{L}^{\prime}\right)$ is sufficient for one-one $(\mathrm{B})$.

For, by definition of $M, f(b+f(a+1)-f(b+f(a) \leqq M(f(b+1)-f(b))$ and $c+f(b+f(a+1)-(c+f(b+f(a)) \leqq M((c+f(b+1)-(c+f(b))$. Now use $\left(\mathrm{L}^{\prime}\right)$ on the interval $(c+f(b), c+f(b+1))$ and we have

$$
\begin{aligned}
f(c+f(b+f(a+1)-f(c+f(b+f(a) & \leqq k M(f(c+f(b+1)-f(c+f(b)) \\
& \leqq k M^{2}(f(c+1)-f(c)) .
\end{aligned}
$$

By iteration of this process, one obtains

$$
\begin{aligned}
f\left(c_{1}+\cdots+f\left(c_{\nu}+1\right)-\right. & f\left(c_{1}+\cdots+f\left(c_{\nu}\right)\right. \\
& \leqq k^{\nu-2} M^{\nu-1}\left(f\left(c_{1}+1\right)-f\left(c_{1}\right)\right) \leqq(k M)^{\nu-2} M,
\end{aligned}
$$

which approaches zero since $k<1 / M$.

While this discussion is cumbersome, it nevertheless shows that a function $f(t)$ defined arbitrarily (consistent with monotonicity) at $t=0,1,2, \cdots, p-1, p$, and then at all $t=b+f(a), a, b$ on $0, \cdots, p-1$, and elsewhere by the broken line connecting these points, must satisfy (L) with $k=1$, since the ratio of inner to outer slope on the straight segments is unity.

Thus the broken line function connecting $f(0)=0, f(1)=e>0$, ( $e$ arbitrarily small constant), $f(1+e)=1-e, f(2)=1$ (for $p=2$ ) yields a one-one $(\mathrm{B})$. The slope on $(1,1+e)$ however is $(1-2 e) / e$, which may be arbitrarily large.

9. Algebraic examples of the one-one case. Example 1. Let $f(t)=t^{n} / p^{n}$ for an integer $p \geqq 2$ and an integer $n$ on $1 \leqq n<p$. One verifies the properties of $\$ 2$, and condition $\left(\mathrm{K}^{\prime \prime}\right)$ with $\beta=n / p$. For $n=1$, our theory reduces to the classical decimals with base $p$. In the general case let $q$ be an integer not greater than $p-2$, and let $C^{0}$ be the limit for sequence $\{q, q, \cdots\}$. Then $C^{0}=q+f\left(C^{0}\right)$, and the number $\alpha=C^{0} / p$ satisfies $p \alpha=q+\alpha^{n}$ or $\alpha^{n}-p \alpha+q=0$, where $0 \leqq q / p \leqq \alpha<(q$ $+1) / p \leqq p-1 / p$. Thus the equation $x^{n}-p x+q=0,1 \leqq n<p, 0 \leqq q$ 
$\leqq p-2$, has exactly one real root on $[0,1)$, namely $\alpha=(1 / p)$ $\cdot(q+f(q+f(q+\cdots)$

In particular, for $n=2, p=3, q=1, x^{2}-3 x+1=0, \alpha=\left(3-5^{1 / 2}\right) / 2$ is approximately

$$
(1 / 3) C_{3}^{0}=(1 / 3)\left(1+\frac{1}{9}\left(1+\frac{1}{9}\left(1+\frac{1}{9}\right)^{2}\right)^{2}\right),
$$

with error not greater than $\beta^{3}=(2 / 3)^{3}$.

Example 2. For $f(t)=(1+t)^{1 / n}-1, p=2^{n}-1, n>1$, one has slope on $(0, p)$ not greater than $1 / n$. We consider $\gamma=1+f(q+f(q+\cdots)$ where $0<q \leqq 2^{n}-2$. We have $\gamma=1+f(q+\gamma-1)=1+(q+\gamma)^{1 / n}-1$ or $\gamma^{n}-\gamma-q=0$. Thus, the equation $x^{n}-x-q=0, n>1,0<q \leqq 2^{n}-2$, has only one real root $\gamma$ on $(1,2]$, namely the number $\gamma$ above.

For instance, $n=2, p=3, q=1, x^{2}-x-1=0, \gamma=1+f(1+f(1+\cdots$. The successive $C_{\nu}{ }^{0}$ are $1+f(1)=2^{1 / 2}=\left(1+1^{1 / 2}\right)^{1 / 2}$ (from here on radicals are "nested"), $1+f\left(1+f(1)=\left(1+\left(1+1^{1 / 2}\right)^{1 / 2}\right)^{1 / 2}\right.$, and so on. Hence $\left(1+5^{1 / 2}\right) / 2=\left(1+\left(1+(1+\cdots)^{1 / 2}\right.\right.$.

Recalling the remark at the end of $\S 4$, and using $n=2, p=3, q=2$, $x^{2}-x-2=0, \gamma=2=1+f(2+f(2+\cdots)$, the successive approximations being $1+f(2)=3^{1 / 2}, 1+f\left(2+f(2)=\left(2+3^{1 / 2}\right)^{1 / 2}, 1+f 2+f 2+f 2\right.$ $=\left(2+\left(2+3^{1 / 2}\right)^{1 / 2}\right)^{1 / 2}$, and so on. But using the sequence $p-1$ $+f(p-1+\cdots+f(p-2)$, we have $3=2+f 2+f 2+\cdots$ with approximations $2+f(1)=1+2^{1 / 2}, 2+f 2+f 1=1+\left(2+2^{1 / 2}\right)^{1 / 2}$, whence $2=\left(2+(2+(2+\cdots))^{1 / 2}\right.$ which is the classical limit occurring in the inscribed polygon theory [2].

Finally, for $n=3, p=2^{n}-1=7, q=6, x^{3}-x-6=0, \gamma=2=1+f(6$ $+f\left(6+\cdots\right.$, the approximations being $1+f 6=7^{1 / 3}, 1+f 6+f 6$ $=\left[6+7^{1 / 3}\right]^{1 / 3}$, or again using the $(p-2)$-terminating approximations, $2=\left\{6+\left[6+(6+\cdots)^{1 / 3}\right]^{1 / 3}\right\}^{1 / 3}$.

10. "Spectra" of the topological maps of the unit interval. Let $T=\left\{F_{1}(t)\right\}$ be the class of all continuous increasing functions on $0 \leqq t \leqq 1$ with $F_{1}(0)=0, F_{1}(1)=1$. These are the topological mappings of the unit interval onto itself [5]. If $p$ is any integer not less than 2 and $f(t)$ is of the type in $\$ 2$ :

(M) $f(t)$ continuous increasing on $0 \leqq t \leqq p ; f(0)=0, f(p)=1$, then $F(t)=f(p t)$ is in the class $T$. Thus all our functions may be regarded as magnifications of the functions of $T$ by a factor $p$ in the $t$-direction. Conversely, if $F_{1}(t)$ is in $T$ and $p \geqq 2$, then $F_{p}(t)=F_{1}(t / p)$ is a function of type (M). Hence for every $F_{1}(t)$ in $T$ we regard the sequence of functions $\left\{F_{1}(t), F_{2}(t), F_{3}(t), \cdots\right\}$ where $F_{p}(t)=F_{1}(t / p)$ for $p \geqq 2$. The associated sequence of perfect sets $L_{p}$ of limit numbers of $F_{p}(t)$ is a curious sort of "spectrum" for $F_{1}$. 
For a fixed $F_{1}(t)$ in $T$, the correspondence $\left(t, F_{1}(t)\right) \leftrightarrow\left(n t, F_{1}(t)\right)$, $0 \leqq t \leqq 1$, is a one-one correspondence of the points on the curves $y=F_{1}(t)$ and $y=F_{n}(t)$. This induces a one-one correspondence between the points of the curves $F_{n}$ and $F_{n+1}$, namely, $\left(n t, F_{1}(t)\right)$ $\leftrightarrow\left((n+1) t, F_{1}(t)\right), 0 \leqq t \leqq 1$. The latter may be used to show that the slopes $s_{n}, s_{n+1}$ of the chords at corresponding points of $F_{n}$ and $F_{n+1}$ satisfy $s_{n}>s_{n+1}$. Hence if some $F_{n}$ satisfies $\left(\mathrm{K}^{\prime}\right)$ so do all succeeding $F_{n}$, and thus $L_{p}=[0, p), p \geqq n$. If $F_{1}$ is of bounded slope, there will exist an $F_{n}$ of slope everywhere less than one. Moreover, one can show that if $F_{n}$ satisfies $\left(\mathrm{D}^{\prime}\right)$ and $\left(\mathrm{J}^{\prime}\right)$, so does $F_{n+1}$. This leads to the question whether $L_{p}=[0, p)$ implies $L_{p+1}=[0, p+1)$. This is in fact not the case.

Example. The broken line function $F_{1}$ defined by $F_{1}(0)=0$, $F_{1}(4 / 9)=1 / 3, F_{1}(5 / 9)=2 / 3, F_{1}(1)=1$ has $L_{2}=[0,2)$, since the product of every two connected slopes of $F_{2}$ is less than one (condition (K) with $n=2$; note that the test (L) fails). But $F_{3}(t)$ is the function of $\$ 7$ with $L_{3}$ of measure zero. However $L_{p}=[0, p), p \geqq 4$, since the maximum slope of $F_{3}$ is one and all successors therefore have slope less than one.

11. Unsolved problems. (1) State simple necessary and sufficient conditions on $f(t)$ such that (B) be one-one. (2) Do there exist functions $f(t)$ which give $C^{0}<\Gamma^{0}$ for non-terminally-periodic sequences $\left\{c_{\nu}\right\}$ ? (3) Do functions exist with sets $L$ of every measure between 0 and $p$ ? (4) The limits of periodic sequences of period $k$ are algebraic numbers of degree $n^{k}$ at most for the function of Example 1, §9. Characterize algebraically all such limits.

\section{REFERENCES}

1. B. H. Bissinger, A generalization of continued fractions, Bull. Amer. Math. Soc. vol. 50 (1944) pp. 868-876.

2. R. Courant and H. Robbins, What is mathematics? Oxford University Press, New York, 1941, p. 125.

3. E. W. Hobson, Theory of functions of a real variable, vol. 1, 3d ed., Cambridge University Press, 1927, p. $117 \mathrm{ff}$.

4. O. Perron, Irrationalzahlen, Berlin, 1921, p. $90 \mathrm{ff}$.

5. J. Schreier and S. Ulam, Eine Bemerkung ïber die Gruppe der topologischen Abbildungen der Kreislinie auf sich selbst, Studia Mathematica vol. 5 (1935) pp. 156-159.

UNIVERSITY OF Wisconsin 